\title{
Regulatory genes of anthocyanins biosynthesis in the barley grain
}

K.V. Strygina ${ }^{1 *}$, E.K. Khlestkina ${ }^{1,2}$

${ }^{1}$ Institute of Cytology and Genetics SB RAS, Novosibirsk, Russia

${ }^{2}$ Novosibirsk State University, Novosibirsk, Russia

*e-mail:pushpandzhali@bionet.nsc.ru

Key words: gene divergence, gene duplication, Hordeum, flavonoids biosynthesis, MBW, transcription factor

Motivation and Aim: Many higher plants including an important agricultural crop barley (Hordeum vulgare L.) synthesize secondary metabolites flavonoids. For activation of the structural flavonoid biosynthesis genes the expression of genes coding transcription transcription factors MYC, MYB and WD40 (forming the MBW regulatory complex) is necessary. The aim of this research was the identification, comparison and analysis of full-length sequences of duplicated MBW gene copies.

Methods and Algorithms: The search of homologous sequences was made in databases for not annotated barley sequences using BLAST. Cluster analysis using MEGA software was based on the UPGMA algorithm. Promoters of the genes were analyzed with PLACE. Primers design for PCR, RT-PCR, qRT-PCR and sequencing was performed using OLIGO. Genetic mapping was performed in DOMxREC mapping population using MAPMAKER program.

Results: Two MYC genes, three MYB genes and two WD40 genes were found in barley genome. The MYC-coding gene $M y c 2$ was mapped precisely (tightly linked to marker XBmac186-4H). Exon-intron organization of all identified genes is similar to the structure of another MBW-coding genes in genomes of dicot and monocot plant species. The transcription activity of the detected genes in various parts of plant varied among the copies.

Conclusion: MYB, bHLH and WD40 genes, controlling the accumulation of blue anthocyanins in the aleurone layer, were first discovered. We showed that the main regulator of the blue pigmentation of the aleurone layer is the bHLH-encoding Myc2 gene, localized on the chromosome 4HL. Identified major allele of the gene is common for the German and Siberian barley populations.

Acknowledgements: This study was supported by the Russian Science Foundation (No. 16-14-00086). 